\title{
Anti-Carcinogenic Possessions of Citrus Peel Extracts and Flavonoids: A Review
}

\section{Bakhtawar Shafique, Shahid Mahmood*, Wajiha Saeed and Muhammad Qamrosh Alam}

Institute of Food Science and Nutrition, University of Sargodha, Pakistan

*Corresponding Author: Shahid Mahmood, Institute of Food Science and Nutrition, University of Sargodha, Sargodha, Pakistan.

Received: June 20, 2019; Published: July 24, 2019

DOI: $10.31080 /$ ASNH.2019.03.0378

\begin{abstract}
Researchers and scientists synthesized numerous drugs and medications to treat cancer. These synthesized drugs related with many side reactions could deteriorate the quality of patient's life. Recently, various functional foods containing effective molecular components against cancer has gained much popularity as no side effects are associated with them and these are natural based product could be easily consumed and can left a long lasting health impression on the life of patients. Citrus peel enriched with flavonoids, polymethoxylated flavones and numerous bioactive components demonstrated anti-carcinogenic, anti-tumour, anti-proliferative and anti-inflammatory activities on skin cancer, gastric cancer, prostate cancer, oseophageal tumorigenesis, colon carcinogenesis, colorectal carcinoma cell, human squamous cell carcinoma and against human cancer cell lines. A novel functional natural product named Gold Lotion (GL) was formulated from citrus peel extract to inhibit the inflammation-associated tumorigenesis. Gold Lotion (GL) demonstrated a clear and promising strategy by in vivo analysis and result showed beneficial aspects to treat prostate cancer, skin cancer and colon cancer in mice. Six different orange peel extracts (OPEs) were prepared containing bioactive potential of polymethoxyflavones (PMFs) and hydroxylated PMFs (OH-PMFs). In vitro study of citrus flavonoids effects on the growth of a human squamous cell carcinoma cell line (HTB43) demonstrated the health benefit and anti-cancer effects by examination of four plant flavonoids (taxifolin, nobiletin, quercetin and tangeretin). Moreover, quercetin chalcone (QC) and pH-modified citrus pectin (MCP) are the modified substances of quercetin (a flavonoid) and citrus pectin (a polysaccharide found in the cell wall of plants). Earlier studies indicated that many antitumor properties due to immune stimulation, free radical scavenging, alteration of the mitotic cycle in tumor cells; gene expression modification, anti-angiogenesis activity and apoptosis induction are exhibited by quercetin. Prostate cancer and melanoma metastases are also inhibited by modified citrus pectin (MCP).
\end{abstract}

Keywords: Citrus Peel; Polymethoxylated Flavonoids; OPEs; GL; Anti-Cancer

\section{Introduction}

Cancer is major fatal disease associated with many side effects, usually treated with synthesized drugs and medications. These side effects could be low blood counts (anemia, leucopenia, thrombocytopenia), hair loss, peripheral neuropathy (numbness and tingling of hands and feet), arthralgias and myalgias (pain in the joints and muscles), nausea and vomiting, diarrhea, mouth sores, hypersensitivity reaction (fever, shortness of breath) and blood clot [1].

Suzawa., et al. (2014) reported that Citrus peel enriched with polymethoxylated flavones, flavonoids and bioactive substances associated to treat malignant tumours and neoplasms. Dietary bioactive components are extracted from citrus peel as well as formulated the products to improve the quality of cancer patient's life and to inhibit the inflammation associated tumorigenesis [2].

\section{Flavonoids}

Flavonoids are the polyphenolic molecules containing 15 carbon atoms and appear as two benzene rings that are linked together by a three-carbon chain. They encourage several beneficial health effects. They are regarded as anti-oxidant, anti-viral, anti-carcinogenic, anti-tumour, anti-inflammatory and anti-proliferative [3]. 


\section{Bioactive citrus peel flavonoids}

Citrus peel contained flavonoids and polymethoxylated flavones are considered to be biologically active compounds against carcinogenesis and tumorigenesis. The major citrus peel flavonoids preventing cancer are nobiletin, quercetin, tangeretin, diosmin, hesperidin, apigenin, luteolin and morin as well as many bioactive substances are studied in this review [4].

\section{Flavonoids nobiletin}

Murakami., et al. (2000) consumption of citrus fruits has been proved to be a major cancer preventive measure. Nobiletin is extracted from citrus peel act as a functionally novel and chemopreventive substance in inflammation correlated tumorigenesis. The processes of epithelial carcinogenesis are directly related with the generation of nitric oxide (NO). NO production inhibitors in Citrus unshiu have been searched through various methods. An activityguiding separation is the technique which was utilized to trace the vigorous substance. Furthermore, the combination of lipopolysaccharide and IFN-gamma with genetic makeup (RAW 264.7 cells) is used to induce the $\mathrm{NO}$ and superoxide $\mathrm{O}_{2}$ generation in mouse macrophage, as well as 12-O-tetradecanoylphorbol-13-acetate (TPA) in differentiated human promyelocyte HL-60 is also involved. The production of $\mathrm{NO}$ and $\mathrm{O}_{2}$ is intimately coupled with epithelial carcinogenesis. Western blotting is a technique through which expression of NO synthase and cycloxygenase 2 were detected. The in-vivo practices and methods are done to evaluate the antitumor and anti-inflammatory promoting activities by applying TPA to ICR mouth skin with several expressions of disease measurements. Nobiletin, a polymethoxyflavonoid inhibited the $\mathrm{NO}$ and $\mathrm{O}_{2}$ generation and also suppressed the two stages of skin inflammation which is induced by applying double TPA [5].

Yoshimizu., et al. (2004) reported that citrus variety depressa hayata was utilized to extract nobiletin which is the citrus flavonoid and demonstrated the effects to avoid and prevent cancer and tumorigenesis Experiments revealed its effect on gastric cell lines TMK-1, MKN-45, MKN-74 and KATO-III. The action of nobiletin on these cells through various ways was analyzed by 3-(4, 5-dimethythiazol-2-yl)-2, 5-diphenyltetrazolium bromide (MTT) assay, the TdT-mediated dUTP biotin nick-end labeling (TUNEL) method and cell-cycle analysis. The nobiletin acted by direct cytotoxicity, modulation of cell cycle and induction of apoptosis. Nobiletin efficacy by the combined treatment with unadventurous anticancer drug,
CDDP was also analyzed as well as examined. To show the synergistic effect compared to the control, CDDP administration was made $24 \mathrm{~h}$ prior to demonstrate its treatment with nobiletin. The bioactive compound nobiletin showed a promising strategy for further investigation and through study nobiletin demonstrated the treatment of gastric cancer [6].

Kohno., et al. (2001) reported that polymethoxyflavonoid nobiletin was directly fed to male F344 rats, separated from citrus unshiu to demonstrate the modifying effects on the growth of azoxymethane (AOM)-induced colonic aberrant crypt foci (ACF). Monoclonal antibody MIB-5 was utilized to observe the effects of nobiletin on cell proliferation activity of ACF. Rats were provided with the subcutaneous injections of AOM (15 mg/kg body weight) once a week for 3 weeks to encourage ACF. They also consumed the experimental diet starting ne week before the first dosing of AOM containing $0.01 \%$ or $0.05 \%$ nobiletin for 5 week. AOM introduction developed $139 \pm 35 \mathrm{ACF} / \mathrm{rat}$ at the end of the study (week 5). Dietary management of nobiletin caused significant reduction in the frequency of ACF: $70 \pm 15$ (50\% reduction, $p, 0.001)$ at a dose of $0.01 \%$ and $63 \pm 10$ (55\% reduction, p,0.001) at a dose of $0.05 \%$. Nobiletin dietary administration significantly lowered MIB-5-index in ACF, decreased prostaglandin E2 content in the colonic mucosa, developed chemopreventive ability by the reduction of cell proliferating activity of ACF development [7].

Lin., et al. (2003) reported that in the mouse macrophage J774A.1 cell line and synovial human fibroblasts, nobiletin ability studied to develop cyclooxygenases (COXs), prostaglandin (PG) $\mathrm{E}_{2}$, and proinflammatory cytokines. In human synovial cells in a dosedependent manner $(<6 \mu \mathrm{M})$, interleukin (IL)-1-induced development of $\mathrm{PGE}_{2}$ was suppressed by Nobiletin. Furthermore, COX-2, the IL-1-induced gene expression and development of proMMP-1/ procollagenase-1 and proMMP-3/ prostromelysin-1 in human synovial fibroblasts was downregulated by nobiletin and also interfered with the lipopolysaccharide-induced development of PGE2 and the gene expression of proinflammatory cytokines comprising IL- $\alpha$, IL-1 $\beta$, TNF- $\alpha$ and IL- 6 in mouse J774A. 1 macrophages. The development of the endogenous MMP inhibitor and the upregulation of TIMP-1 encouragement is a novel action of nobiletin [8].

Flavonoids diosmin and hesperidin

Tanaka., et al. (1997) reported that the dietary feeding of two flavonoids, diosmin and hesperidin initiated with azoxymethane 
(AOM) to inhibit colon carcinogenesis utilized in combination during the initiation phases to investigate and analysis of cure effects experimented in male F344 rates. The diets of rats comprised of feeding at different concentration, containing diosmin (1000 ppm), hesperidin (1000 ppm) or diosmin (900 ppm) and hesperidin (100 ppm) for 5 weeks during initiation treatment or 28 weeks for post-initiation treatment. AOM was weekly injected at the dose of $15 \mathrm{mg} / \mathrm{kg}$ of body wt which induces colon neoplasms. The study showed that the group of rats which were fed with AOM alone $(\mathrm{P}<0.001)$ have the large effect of multiplicity of neoplasms adenoma and adenocarcinoma) in the large intestine than those group of rats which were treated with the combination of diosmin and hesperidin. The consumption of diosmin and hesperidin used in combination showed the inhibition of development of colonic neoplasm as well as prevention of development of aberrant crypt foci. The 5'-bromodeoxy-uridine-labelling index and argyrophilic nuclear organizer region's number in crypt cells, colonic mucosal ornithine decarboxylase activity, and polyamine levels in the blood could also be reduced by dietary intake of diosmin and hesperidin and act as chemopreventive and antiproliferative agents to inhibit colon carcinogenesis. The result demonstrated no pathological alternations in rats treated with diosmin and hesperidin supplementation, individually or in combination as well as played role in reduction the expression of cell proliferation biomarkers (BrdUlabelling index and AgNORs number) of non-lesional oesophageal epithelium $(\mathrm{P}<0.05)$ and lower the blood polyamine attentiveness, also effective in suppression of oesophageal cancer development and inhibited the suppression of increased cell proliferation caused by MNAN in the oesophageal mucosa [9].

Dietary administration of diosmin and hesperidin

\begin{tabular}{|l|c|c|c|}
\hline Flavonoids & \multicolumn{2}{|c|}{ Doses fed in F344 rats } & Duration \\
\hline & $\begin{array}{c}\text { Initiation } \\
\text { treatment }\end{array}$ & $\begin{array}{c}\text { Post-initiation } \\
\text { treatment }\end{array}$ & \\
\hline Diosmin & $1000 \mathrm{ppm}$ & $1000 \mathrm{ppm}$ & 5 weeks \\
\hline Hesperidin & $900 \mathrm{ppm}$ & $100 \mathrm{ppm}$ & 28 weeks \\
\hline & Doses fed in male wistar rats & \\
\hline & $\begin{array}{c}\text { Initiation } \\
\text { treatment }\end{array}$ & $\begin{array}{c}\text { Post-initiation } \\
\text { treatment }\end{array}$ & \\
\hline Diosmin & $1000 \mathrm{ppm}$ & $900 \mathrm{ppm}$ & 13 weeks \\
\hline Hesperidin & $1000 \mathrm{ppm}$ & $100 \mathrm{ppm}$ & 7 weeks \\
\hline
\end{tabular}

Table a

\section{Flavonoids tangeretin}

Hirano., et al. reported that human tumour cells are treated with various anticancer agents in order to encourage apoptosis. Normal tissue origin cells comprised of myelocytes and immunocytes are fundamentally cytotoxic by the induction of anticancer agents. There was observed no cytotoxicity against human peripheral blood mononuclear cells (PBMCs) by the treatment with tangeretin $\left(5,6,7,8,4^{\prime}\right.$-pentamethoxyflavone) which induces apoptosis in human promyelocytic leukaemia HL-60 cells. Tangeretin IC ${ }_{50}$ value range between 0.062 and $0.173 \mu \mathrm{M}$ was used to suppress the growth of HL-60 cells in vitro evaluated by $\left({ }^{3} \mathrm{H}\right)$ thymidine incorporation or tetrazolium crystal formation. $>2.7 \mu \mathrm{M}$ tangeretin was used to demonstrate the apoptosis of HL-60 cells in vitro evaluated by cell morphology and DNA fragmentation. The treatment of HL60 cells with tangeretin contain low DNA content utilized to demonstrate the apoptotic cells by cytometric analysis and observed a reduction in G1 cells and a affiliated boost of $\mathrm{S}$ and/or G2/M cells. After the 24 hours of application with tangeretin, apoptosis was observed and DNA fragmentation was evaluated the tangeretin effect $\mathrm{Ca}^{2+}$ - dependent endonuclease activity was inhibited by the occurrence of $\mathrm{Zn}^{2+}$ attenuated by growth inhibition. Cycloheximide drastically caused reduction in the tangeretin effect on HL-60 cell growth. Dye exclusion test demonstrated that at higher concentration $(27 \mu \mathrm{M})$ no cytotoxicity was observed by the application of tangeretin against HL-60 cells or mitogen-activated PBMCs. Furthermore, the flavonoid was ineffectual on growth of human T-lymphocytic leukaemia MOLT-4 cells or on blastogenesis of PBMCs. The result evident that tangeretin decreases the growth of HL-60 cells in vitro, moderately through apoptosis induction, devoid of severe side-effects on immune cells [10].

Pan., et al. 2002 reported that citrus peel is rich in tangeretin which is 5,6,7,8,4-pentamethoxyflavone that indicated through DNA flow cytometric analysis, cell cycle progression was blocked with tangeretin. Tangeretin demonstrated this effect in colorectal carcinoma COLO 205 cells at $G_{1}$ phase. The development of $G_{1}$ arrest and decrease in the degree of phosphorylation of $\mathrm{Rb}$ was experienced over a $24 \mathrm{~h}$ exposure to tangeretin. It is showed that there is a reduction in the protein expression of cyclins A, D1 and E slightly under the same conditions. Immunocomplex kinase experiments showed that tangeretin prevented the cyclin-dependent kinase 2 (Cdk2) and 4 (Cdk4) in the cell-free system in a dose dependent 
manner. It is occurred in the loss of both Cdk2 and 4 kinase activities gradually when cells were exposed to tangeretin $(50 \mu \mathrm{M})$ over 48h. the content of the Cdk inhibitor p21 protein was increased by tangeretin related with the p53 levels elevation. Moreover, within $18 \mathrm{~h}$, there is a increase in the level of Cdk inhibitor p27 protein by tangeretin. It is suggested through results that the growth-inhibitory effects through modulation of the activities of several key G1 regulatory proteins, such as $\mathrm{Cdk} 2$ and $\mathrm{Cdk} 4$ were exerted through tangeretin and it also mediates the increase of Cdk inhibitors p21 and p27 [11].

\section{Gold lotion}

Pan., et al. (2012) reported that a novel functional natural product named Gold Lotion (GL) was formulated from citrus peel extract to inhibit the inflammation-associated tumorigenesis. It is reported by experiment and analysis, Gold Lotion has inhibitory effects on 12-O-tetradecanoylphorbol 13-acetate (TPA)-induced expression of inducible nitric oxide synthase (iNOS) and cyclooxygenase-2 (COX-2) in mouse skin. The idea is obtained from TPA-induced skin inflammation model, in mouse skin the transcriptional activation iNOS and its mRNA and protein has been efficiently inhibited by the topical application of Gold lotion. It is found that, by decreasing inflammatory gene parameters the GL effectively prevented TPA-induced inflammation in mouse skin. Moreover, GL significantly repressed 7,12-dimethylbenez[a]anthracene (DMBA)/ TPA-induced skin tumor development and prevented tumor occurrence, tumor influence and tumor diversity of papillomas at 20 weeks. These in vivo data have discovered that GL is an effectual anti-tumor agent that assists by down-regulating the protein levels of COX-2, ornithine decarboxylase (ODC), and vascular endothelial growth factor (VEGF) in mouse skin. Peels of Citrus genus comprised of polymethoxyflavonoids (PMFs), predominantly they are found in the peels of sweet oranges (Citrus sinensis) and mandarin (Citrus reticulate). Gold lotion (GL) is formed from the peels of 6 citrus fruits named as navel oranges, citrus hassaku, citruslimon, citrus natsudaidai, citrus miyauchi and satsuma accessible in Japan. GL was mainly developed as a cosmetic product to defend skin from UV irradiation. Patrons later reported subjective indication that depend on the topical application of GL to avoid melanoma, GL contained anti-cancer property and oral intake for prostate, lung and liver cancers. The efficacy of GL has the inhibitory possessions on TPA-induced iNOS, COX-2, and ODC expression in mouse skin; evaluated the anti-inflammatory activity of GL in mouse skin fol- lowing TPA application was investigated and the inhibitory effect of GL on mouse skin tumorigenesis using a two-stage skin carcinogenesis model by the assessment of tumor occurrence, diversity and volume has been explored [12].

Suzawa., et al. (2014) reported that humans are facing two major lethal diseases, cancer is one of them. Researchers and scientists synthesized numerous drugs and medications but they are also related with many side reactions. These synthesized drugs can deteriorate the quality of patient's life. Lately, various functional foods containing effective molecular components against cancer has gained much popularity as no side effects are associated with them and these are natural based product could be easily consumed and can left a long lasting health impression on the life of patients. Citrus peels are very effective associated with numerous polymethoxyflavones and flavonoids to treat cancer. It has been reported that citrus peel extract contain health benefit and regarded to be as beneficial as pharmacological products. The overall results of these studies have proved to be effective by experiments and analysis and Gold Lotion (GL) was prepared. Various varieties of citrus peels were experimented and after the analysis of results, a effective health beneficial product Gold Lotion associated with the extracts of polymethoxyflavonoids was formulated. Gold Lotion (GL) demonstrated a clear and promising strategy by in vivo analysis and result showed beneficial aspects to treat prostate cancer, skin cancer and colon cancer in mice. The prevention and treatment of cancer by gold lotion has proved to be a access clinical trials and also demonstrated opportunities for the various researches in near future [13].

Effects of GL administration on the body weight and organ weight in a PC-3 xenograft model

Lai., et al. (2013) reported that prostate cancer is the most prevailing lethal disease among humans could be inhibited by the varieties of citrus peel extracts and flavonoids. Azoxymethane (AOM) induced colon tumorigenesis could be prevented by polymethoxyflavonoids obtained from citrus peel found in formulated product Gold lotion (GL). Human prostate xenograft tumor mouse model was utilized to predict the efficacy of Gold lotion (GL). GL caused the reduction in both weight (57-100\% reduction) and volume (78$94 \%$ reduction) in tumour by both intraperitoneal injection and oral examination. Moreover, GL demonstrated down regulation of the protein levels of inflammatory enzymes (inducible nitric oxide 
synthase, iNOS and cyclooxygenase-2, COX-2), metastasis (matrix metallopeptidase-2, MMP-2 and MMP-9), angiogenesis (vascular endothelial growth factor, VEGF), and proliferative molecules, as well as by the induction of apoptosis in prostate tumors [14].

\begin{tabular}{|c|c|c|c|c|c|c|}
\hline Treatment & Group & No. of mice & Body weight (g) & Liver (mg) & Spleen (mg) & Lung (mg) \\
\hline \multirow[t]{3}{*}{ i.p. } & control & 8 & $16.17 \pm 4.90$ & $0.91 \pm 0.35$ & $0.07 \pm 0.04$ & $0.09 \pm 0.01$ \\
\hline & GL $1 \mathrm{mg} \mathrm{kg}^{-1}$ & 6 & $16.36 \pm 1.77$ & $0.83 \pm 0.07$ & $0.05 \pm 0.01$ & $0.13 \pm 0.06$ \\
\hline & GL $2 \mathrm{mg} \mathrm{kg}^{-1}$ & 6 & $23.18 \pm 0.83$ & $1.49 \pm 0.07$ & $0.07 \pm 0.04$ & $0.12 \pm 0.02$ \\
\hline \multirow[t]{3}{*}{ Oral } & control & 8 & $20.41 \pm 2.11$ & $1.15 \pm 0.12$ & $0.08 \pm 0.02$ & $0.11 \pm 0.02$ \\
\hline & GL $2 \mathrm{mg} \mathrm{kg}^{-1}$ & 6 & $21.22 \pm 0.53$ & $1.06 \pm 0.09$ & $0.09 \pm 0.02$ & $0.13 \pm 0.08$ \\
\hline & GL $4 \mathrm{mg} \mathrm{kg}^{-1}$ & 6 & $21.34 \pm 0.90$ & $1.33 \pm 0.21$ & $0.08 \pm 0.03$ & $0.13 \pm 0.07$ \\
\hline
\end{tabular}

Table b

\section{Orange peel extracts}

Gosslau., et al. (2014) reported that six different orange peel extracts (OPEs) were prepared containing bioactive potential of polymethoxyflavones (PMFs) and hydroxylated PMFs (OH-PMFs). The characterization and quantification of these peel extracts were done with the help of high performance liquid chromatography (HPLC). Utilizing a human cell-based TPA-induced monocytemacrophage differentiation model which employs U-937 cells and inflammatory surrogate gene leads to the analysis of effects on inflammation by nutrigenomics. Dose response and kinetics analysis of OPEs with dissimilar chemical profiles exposed less cytotoxic effects of PMFs as compared to OH-PMFs as established by the MTT-method. It is worth mentioning here, a evaluation of two PMF members such as 3,5,6,7,3_4_-hexamethoxyflavone (HexaMF) and 3,5,6,7,8,3_4_-heptamethoxyflavone (HeptaMF) lasted less cytotoxic effects of HeptaMF as compared to HexaMF. A particular OPE enriched with HeptaMF, PMFs and OH-PMFs at low concentrations (10 _ g/mL)appreciably down-regulated the appearance of a group of genes implicated in inflammatory rejoinder, together with $C O X-2, T N F-\alpha, I C A M-1, N F \kappa B, I L-1 \beta, I L-6$, and $I L-8$ with an provocative catalog of -0.55 .The strapping anti-inflammatory effects were then validated in a mouse carrageenan-induced paw edema model. Oral ingestion of OPE reduced paw edema drastically in a dose-dependent approach. A quantity of $250 \mathrm{mg} / \mathrm{kg}$ gavean has anti-inflammatory effect as compared to ibuprofen. A preface clinical study revealed that OPE was well tolerated showing no unsympathetic adverse reactions. Fortification of phyto extracts such as OPEs with definite polymethoxyflavones as anti-inflammatory bioactives is a gifted stratagem to find unsurprisingly derivative extracts that are effectual in opposition to ailments coupled with inflammation [15].
Lai., et al. (2011) reported that the molecular mechanism and chemopreventive effects of dietary administration of 0.01 and 0.05\% hydroxylated PMFs in AOM-induced colonic tumorigenesis were investigated by institute of cancer research (ICR) in male mice at the age of 6 week twice weekly dose of $5 \mathrm{mg} / \mathrm{kg}$ for 2 week. At 6 and 20 week, colonic tissues were collected from mouse. Dosedependent dietary administration of hydroxylated PMFs feeding caused reduction in the number of aberrant crypt foci and tumours in colonic tissues, reduced the levels of inducible nitric oxide synthase, cyclooxygenase, cyclin D1 and vascular endothelial growth factor through interfering with Wnt/b-catenin and epidermal growth factor receptor/Ras/mitogen activated protein kinase signaling pathways as well as the activation of transcription factors NF-kB and STAT3 in colonic tissue by in vivo analysis [16].

Composition and contents of PMF and hydroxylated PMF in orange peel extract

\begin{tabular}{|c|c|}
\hline OH-PMFs and PMFs & $\begin{array}{l}\text { Concentration } \\
\text { (mg/g OH-PMFs) }\end{array}$ \\
\hline 5-Hydroxy-3,6,7,8,3',4'hexamethoxyflavone & $254.78 \pm 4.95$ \\
\hline 5-Hydroxy-6,7,8,3',4'-pentamethoxyflavone & $396.42 \pm 4.62$ \\
\hline 5-hydroxy-3,6,7,3',4'-pentamethoxyflavone & $7.08 \pm 0.11$ \\
\hline 5-hydroxy-6,7,8,4'-tetramethoxyflavone & $74.96 \pm 0.16$ \\
\hline 5-hydroxy-6,7,4'-trimethoxyflavone & $115.52 \pm 2.35$ \\
\hline 5-hydroxy-6,7,3',4'-tetramethoxyflavone & $44.5 \pm 0.08$ \\
\hline Nobiletin & $10.41 \pm 0.14$ \\
\hline Heptamethoxyflavone & $55.04 \pm 0.46$ \\
\hline Tangeretin & $20.34 \pm 0.62$ \\
\hline
\end{tabular}

Table c 
Effects of dietary hydroxylated PMFs on AOM-induced ACF formation in ICR mice

\begin{tabular}{|l|c|c|c|c|c|}
\hline Group & $\begin{array}{c}\text { No. of } \\
\text { mice }\end{array}$ & $\begin{array}{c}\text { Body weight } \\
\text { (g) }\end{array}$ & $\begin{array}{c}\text { No.ACF/colon } \\
\text { ACF }\end{array}$ & Large ACF & $\begin{array}{c}\text { Incidence of } \\
\text { ACF formation }\end{array}$ \\
\hline AOM & 15 & $37.9 \pm 2.3$ & $47 \pm 3^{\text {b) }}$ & $23 \pm 3^{\text {b) }}$ & $15 / 15(100 \%)$ \\
\hline AOM + 0.01\% hydroxylated PMFs & 15 & $38.4 \pm 2.4$ & $29 \pm 2^{\text {b) }}$ & $13 \pm 4^{\text {b) }}$ & $15 / 15(100 \%)$ \\
\hline AOM + 0.05\% hydroxylated PMFs & 15 & $38.5 \pm 2.9$ & $29 \pm 6^{\text {b) }}$ & $10 \pm 2^{\text {b) }}$ & $15 / 15(100 \%)$ \\
\hline
\end{tabular}

Table d

a) All mice of each group were killed by decapitation at the end of wk 6 . The colons were removed and fixed in $10 \%$ buffered

formalin. ACF in formalin-fixed colons were identified as crypts with increased methylene blue staining and expanded pericryptal spaces ( $n=15$ ). The average number of ACF and large ACF ( $\geq 6$ component crypts/focus) were expressed as mean \pm SE per colon.

b) $p<0.01$, compared with AOM-treated alone.

Effects of dietary hydroxylated PMFs on the AOM-induced colonic tumor in male ICR mice

\begin{tabular}{|l|c|c|c|c|c|c|}
\hline Group & $\begin{array}{c}\text { No. of } \\
\text { mice }\end{array}$ & $\begin{array}{c}\text { Body weight } \\
\text { (g) }\end{array}$ & Liver (mg) & Spleen (mg) & $\begin{array}{c}\text { Tumor } \\
\text { multiplicity }\end{array}$ & $\begin{array}{c}\text { Tumor } \\
\text { incidence (\%) }\end{array}$ \\
\hline AOM & 15 & $46.6 \pm 3.2$ & $2.5 \pm 0.4$ & $0.22 \pm 0.04$ & $7.8 \pm 1.3^{\text {b) }}$ & $15 / 15(100 \%)$ \\
\hline AOM + 0.01\% hydroxylated PMFs & 15 & $40.6 \pm 3.2$ & $2.1 \pm 0.4$ & $0.23 \pm 0.01$ & $4.0 \pm 0.8^{\text {c })}$ & $15 / 15(100 \%)$ \\
\hline AOM + 0.05\% hydroxylated PMFs & 15 & $42.4 \pm 4.2$ & $2.2 \pm 0.3$ & $0.23 \pm 0.02$ & $1.5 \pm 0.6^{\text {b) }}$ & $15 / 15(100 \%)$ \\
\hline
\end{tabular}

Table e

a) All mice of each group were killed by decapitation at the end of week 20. Colon tissues were analyzed by H\&E stain for microadneoma. The average numbers of tumor were expressed as mean \pm SE per colon.

b) $P<0.01$, compared with AOM-treated alone.

c) $P<0.01$, compared with AOM-treated alone.

The data and analysis result demonstrated that dietary administration of hydroxylated PMFs treatment inhibited ACF formation, inhibited AOM induced iNOS and COX-2 expression through downregulating NF-kB and STAT3 signaling, prevent the AOM-upregulated EGFR and Ras signaling, inhibited the AOM induce colonic tumor formation.

\section{Flavonoids quercetin}

Hayashi., et al. (2000) reported that quercetin chalcone (QC) and $\mathrm{pH}$-modified citrus pectin (MCP) are the modified substances of quercetin (a flavonoid) and citrus pectin (a polysaccharide found in the cell wall of plants). Earlier studies indicated that many antitumor properties due to immune stimulation, free radical scav- enging, alteration of the mitotic cycle in tumor cells; gene expression modification, anti-angiogenesis activity and apoptosis induction are exhibited by quercetin. Prostate cancer and melanoma metastases has inhibited by MCP. The result examined the effects of MCP and QC on the size and weight of colon-25 tumors implanted in balb-c mice. Fifty mice were orally administered either $1 \mathrm{~mL}$ distilled water (controls), low-dose QC $(0.8 \mathrm{mg} / \mathrm{mL})$, high-dose QC (1.6 mg/mL), low-dose MCP (0.8 mg/mL) or high-dose MCP (1.6 $\mathrm{mg} / \mathrm{mL}$ ) on a daily basis, beginning the first day of tumor palpation (usually eight days post-implantation). A significant reduction in tumor size was observed at day 20 in all groups compared to controls. The groups given low-dose QC and MCP had a 29-percent 
(NS) and 38-percent $(\mathrm{p}<0.02)$ decrease in size, respectively. The high-dose groups had an even more impressive reduction in size; 65 percent in the QC group and 70 percent in the mice given MCP (both $\mathrm{p}<0.001$ ). MCP demonstrated the reduction in the growth of solid primary tumors, and QC has antitumor activity [17]

Relationship of citrus peel flavonoids to human cancers

Manthey., et al. (2000) reported that numerous types of phenols which are mainly comprised of hydroxycinnamates, flavonoid glycosides, and polymethoxylated flavones are found in rich quan- tity in citrus fruits as well as peel. Proliferation of a number of cancer cell lines is prevented by polymethoxylated flavones which are devoid of glycosidic linkages. Strong antiproliferative action was exhibited by synthetic methoxylated flavones which are proved to act similar activities and action as naturally found compounds. Experiments showed in various cases 10 micrometer occurred above IC50 values. It is demonstrated by various experiments, analysis and results that these polymethoxylated flavones, including hydroxylated flavones and flavanone exhibited antiproliferated as well as anticancer agents in humans [18].

\begin{tabular}{|c|c|c|c|c|c|c|c|}
\hline Compound & $\mu \mathrm{m}$ & Lung & Colon & Breast ER- & Breast ER+ & Prostate & Melanoma \\
\hline \multirow[t]{2}{*}{ (1) $3,5,6,7,8,3^{\prime}, 4^{\prime}$-heptamethoxyflavone } & $\mathrm{IC}_{50}$ & 4.6 & 2.1 & 0.9 & 0.2 & 1.8 & 9.9 \\
\hline & $\mathrm{IC}_{90}$ & 14.5 & 8.8 & 2.8 & 2.3 & 7.4 & 16.4 \\
\hline \multirow[t]{2}{*}{ (2) tangeretin } & $\mathrm{IC}_{50}$ & 3.2 & 1.6 & 1.3 & 0.34 & 0.54 & 0.27 \\
\hline & $\mathrm{IC}_{90}$ & 6.7 & 4.0 & 3.5 & 2.7 & 2.7 & 3.8 \\
\hline \multirow[t]{2}{*}{ (3) nobiletin } & $\mathrm{IC}_{50}$ & 3.5 & 4.7 & 1.2 & 2.9 & 1.0 & 0.50 \\
\hline & $\mathrm{IC}_{90}$ & 10.1 & 13.9 & 3.7 & 6.2 & 2.2 & 2.0 \\
\hline \multirow[t]{2}{*}{ (4) sinensetin } & $\mathrm{IC}_{50}$ & 13.7 & 9.5 & 3.9 & 5.5 & 16.5 & 10.8 \\
\hline & $\mathrm{IC}_{90}$ & 23.6 & 13.9 & 7.4 & 9.7 & 22.5 & 17.9 \\
\hline \multirow[t]{2}{*}{ (5) tetra- $O$-methylscutellarein } & $\mathrm{IC}_{50}$ & 21.5 & 6.3 & 0.80 & 0.53 & 3.9 & 2.9 \\
\hline & $\mathrm{IC}_{90}$ & 39.4 & 13.7 & 2.9 & 2.4 & 8.1 & 7.6 \\
\hline \multirow[t]{2}{*}{ (6) 5-desmethylnobiletin } & $\mathrm{IC}_{50}$ & 38.8 & 8.5 & 0.77 & 0.23 & 2.8 & 3.6 \\
\hline & $\mathrm{IC}_{90}$ & 143 & 171 & 2.6 & 0.77 & 162 & 92 \\
\hline \multirow[t]{2}{*}{ (7) tetra-0-methylisoscutellarein } & $\mathrm{IC}_{50}$ & 18.1 & 6.6 & $\mathrm{ND}^{\mathrm{a}}$ & ND & 2.6 & 11.3 \\
\hline & $\mathrm{IC}_{90}$ & 25.8 & 19.7 & ND & ND & 11.5 & 19.7 \\
\hline \multirow[t]{2}{*}{ (8) 5-desmethylsinensetin } & $\mathrm{IC}_{50}$ & 0.11 & 5.0 & 0.06 & 0.03 & 2.2 & 1.1 \\
\hline & $\mathrm{IC}_{90}$ & 1.7 & 9.5 & 1.7 & 1.1 & 7.8 & 4.7 \\
\hline \multirow{2}{*}{$\begin{array}{l}\text { (9) quercetin } 3,5,7,3^{\prime}, 4^{\prime} \text { - pentamethyl } \\
\text { ether }\end{array}$} & $\mathrm{IC}_{50}$ & 2.2 & 33 & 19 & 18 & 6.0 & 12.1 \\
\hline & $\mathrm{IC}_{90}$ & 5.5 & 110 & 59 & 57 & 74 & 45 \\
\hline \multirow{2}{*}{$\begin{array}{l}\text { (10) quercetin } 3,7,3^{\prime}, 4^{\prime} \text { - tetramethyl } \\
\text { ether }\end{array}$} & $\mathrm{IC}_{50}$ & 6.9 & 0.84 & 26 & 14 & 16 & 8.1 \\
\hline & $\mathrm{IC}_{90}$ & 21 & 47 & 54 & 41 & 56 & 42 \\
\hline \multirow{2}{*}{$\begin{array}{l}\text { (11) limocitrin 3,5,7,4'-tetramethyl } \\
\text { ether }\end{array}$} & $\mathrm{IC}_{50}$ & 17 & 45 & 2.0 & 0.50 & 14 & 6.2 \\
\hline & $\mathrm{IC}_{90}$ & 47 & 107 & 7.2 & 4.0 & 97 & 42 \\
\hline \multirow{2}{*}{$\begin{array}{l}\text { (12) quercetin 5,7,3', } 4^{\prime} \text {-tetramethyl } \\
\text { ether }(S) b\end{array}$} & $\mathrm{IC}_{50}$ & 3.1 & 15.6 & 27 & 14.2 & 5.6 & 3.9 \\
\hline & $\mathrm{IC}_{90}$ & 5.9 & 28 & 70 & 64 & 21 & 5.6 \\
\hline \multirow{2}{*}{$\begin{array}{l}\text { (13) limocitrin 3,7,4'-trimethyl ether } \\
\text { (S) }\end{array}$} & $\mathrm{IC}_{50}$ & 6.4 & 54 & 8.0 & 1.3 & 11 & 5.4 \\
\hline & $\mathrm{IC}_{90}$ & 44 & 115 & 21 & 8.2 & 69 & 41 \\
\hline \multirow{2}{*}{$\begin{array}{l}\text { (14) quercetin 5,7,3', } 4^{\prime} \text {-tetramethyl } \\
\text { ether-3-acetate (S) }\end{array}$} & $\mathrm{IC}_{50}$ & 21 & 14 & 8.5 & 3.0 & 20 & 14 \\
\hline & $\mathrm{IC}_{90}$ & 27 & 22 & 20 & 15 & 27 & 21 \\
\hline \multirow{2}{*}{$\begin{array}{l}\text { (15) limocitrin 3,7,4'-trimethyl } \\
\text { ether-5-acetate (S) }\end{array}$} & $\mathrm{IC}_{50}$ & 2.3 & 18 & 2.1 & 0.11 & 2.6 & 5.3 \\
\hline & $\mathrm{IC}_{90}$ & 17 & 69 & 8.0 & 1.6 & 23 & 20 \\
\hline \multirow{2}{*}{$\begin{array}{l}\text { (16) quercetin 3,7,3',4'-tetramethyl } \\
\text { ether-5-acetate (S) }\end{array}$} & $\mathrm{IC}_{50}$ & 15 & 8.8 & ND & ND & 7.0 & 3.0 \\
\hline & $\mathrm{IC}_{90}$ & 18 & 45 & ND & ND & 50 & 6.3 \\
\hline \multirow{2}{*}{$\begin{array}{l}\text { (17) 5,8-dihydroxy-3,7,3', } 4^{\prime} \text { - } \\
\text { tetramethoxyflavone (S) }\end{array}$} & $\mathrm{IC}_{50}$ & 18 & 21 & ND & ND & 41 & 14 \\
\hline & $\mathrm{IC}_{90}$ & 59 & 85 & ND & ND & 152 & 48 \\
\hline
\end{tabular}

Table 1: Antiproliferative Activities $\left(\mathrm{IC}_{50}\right.$ and $\mathrm{IC}_{90}$ ) of Naturally Occurring and Synthetic Analogues of Polymethoxylated Flavones in Citrus against Six Human Cancer Cell Line. 


\begin{tabular}{|c|c|c|c|c|c|}
\hline Compound & $\mu \mathrm{m}$ & lung & colon & prostate & melanoma \\
\hline \multirow[t]{2}{*}{ (1) 3,5,6,7,8,3థ,4\$-heptamethoxyflavone } & $\mathrm{LC}_{50}$ & 48 & 41 & 126 & 34 \\
\hline & $\mathrm{LC}_{90}$ & $>200$ & 124 & $>200$ & 142 \\
\hline \multirow[t]{2}{*}{ (9) quercetin $3,5,7,3 \varangle, 4 \$$-pentamethyl ether } & $\mathrm{LC}_{50}$ & 79 & 47 & 110 & 47 \\
\hline & $\mathrm{LC}_{90}$ & $>200$ & 165 & $>200$ & 191 \\
\hline \multirow[t]{2}{*}{ (10) quercetin $3,7,3 \uparrow, 4 \$$ - tetramethyl ether } & $\mathrm{LC}_{50}$ & 47 & 41 & $>153$ & 56 \\
\hline & $\mathrm{LC}_{90}$ & $>200$ & $>200$ & $>200$ & $>200$ \\
\hline \multirow[t]{2}{*}{ (11) limocitrin 3,5,7,4\$-tetramethyl ether } & $\mathrm{LC}_{50}$ & 50 & 47 & 111 & 47 \\
\hline & $\mathrm{LC}_{90}$ & $>200$ & 133 & $>200$ & 190 \\
\hline \multirow[t]{2}{*}{ (12) quercetin 5,7,3థ,4\$-tetramethyl ether } & $\mathrm{LC}_{50}$ & 6.4 & 7.8 & 117 & 6.9 \\
\hline & $\mathrm{LC}_{90}$ & 44 & 35 & $>200$ & 106 \\
\hline \multirow[t]{2}{*}{ (13) limocitrin 3,7,4\$-trimethyl ether } & $\mathrm{LC}_{50}$ & 85 & 39 & $>100$ & 39 \\
\hline & $\mathrm{LC}_{90}$ & $>200$ & 97 & 180 & $>200$ \\
\hline \multirow[t]{2}{*}{ (15) limocitrin 3,7,4\$-trimethyl ether-5-acetate } & $\mathrm{LC}_{50}$ & 24 & 22 & 104 & 23 \\
\hline & $\mathrm{LC}_{90}$ & 171 & 72 & $>200$ & 123 \\
\hline \multirow{2}{*}{$\begin{array}{l}\text { (16) quercetin 3,7,3థ,4\$-tetramethyl ether- } \\
\text { 5-acetate }\end{array}$} & $\mathrm{LC}_{50}$ & 18 & 13 & 112 & 2.5 \\
\hline & $\mathrm{LC}_{90}$ & $>200$ & 87 & $>200$ & 170 \\
\hline \multirow{2}{*}{$\begin{array}{l}\text { (17) 5,8-dihydroxy 3,7,3థ,4\$-tetramethoxyfla- } \\
\text { vone }\end{array}$} & $\mathrm{LC}_{50}$ & $>200$ & 48 & $>200$ & $>200$ \\
\hline & $\mathrm{LC}_{90}$ & $>200$ & 189 & $>200$ & $>200$ \\
\hline
\end{tabular}

Table 2: Toxicity $\left(\mathrm{LC}_{50^{\prime} 90}\right)$ of Polymethoxylated Flavones toward Six Human Cancer Cell Lines.

\begin{tabular}{|l|l|c|c|c|c|c|}
\hline Compound & $\boldsymbol{\mu m}$ & lung & colon & Breast ER+ & Prostate & melanoma \\
\hline (18) rhamnetin & $\mathrm{IC}_{50}$ & 38 & 76 & 85 & 22 & 25 \\
& $\mathrm{IC}_{90}$ & 174 & $>200$ & $>200$ & 158 & $>200$ \\
\hline 19) kaempferol & $\mathrm{IC}_{50}$ & 115 & 42 & $>200$ & 95 & $>200$ \\
& $\mathrm{IC}_{90}$ & $>200$ & $>200$ & $>200$ & $>200$ & $>200$ \\
\hline \multirow{2}{*}{ (20) chrysoeriol } & $\mathrm{IC}_{50}$ & 17 & 20 & 7 & 30 & 23 \\
& $\mathrm{IC}_{90}$ & 36 & 46 & 20 & 69 & 59 \\
\hline (21) apigenin & $\mathrm{IC}_{50}$ & 41 & 29 & 22 & 37 & 41 \\
& $\mathrm{IC}_{90}$ & 100 & 85 & 85 & 93 & 125 \\
\hline (22) luteolin & $\mathrm{IC}_{50}$ & 3.1 & 10.5 & 21 & 32 & 32 \\
& $\mathrm{IC}_{90}$ & 12 & 28 & 46 & 70 & 77 \\
\hline (23) quercetin & $\mathrm{IC}_{50}$ & 59 & 40 & 73 & 86 & $>200$ \\
& $\mathrm{IC}_{90}$ & 158 & 115 & 168 & 181 & $>200$ \\
\hline
\end{tabular}

Table 3: Antiproliferative Activities $\left(\mathrm{IC}_{50}\right.$ and $\mathrm{IC}_{90}$ ) of Hydroxylated Flavone Aglycons against Five Human Cancer Cell Lines. 


\begin{tabular}{|l|c|c|c|c|c|c|}
\hline compound & $\boldsymbol{\mu m}$ & lung & colon & Breast ER+ & prostate & melanoma \\
\hline \multirow{2}{*}{ (24) eriodictyol } & $\mathrm{IC}_{50}$ & 87 & 62 & 56 & 42 & $>200$ \\
& $\mathrm{IC}_{90}$ & $>200$ & 180 & 156 & 180 & $>200$ \\
\hline \multirow{2}{*}{ (25) hesperetin } & $\mathrm{IC}_{50}$ & 181 & 149 & 181 & 181 & $>200$ \\
& $\mathrm{IC}_{90}$ & $>200$ & $>200$ & $>200$ & $>200$ & $>200$ \\
\hline \multirow{2}{*}{ (26) naringenin } & $\mathrm{IC}_{50}$ & 102 & 154 & 84 & 150 & 77 \\
& $\mathrm{IC}_{90}$ & $>200$ & 180 & $>200$ & $>200$ & 158 \\
\hline
\end{tabular}

Table 4: Antiproliferative Activities $\left(\mathrm{IC}_{50^{\prime} 90}\right.$ ) of Naturally Occurring Hydroxylated Flavanone Aglycons against Five Human Cancer Cell Lines.

\begin{tabular}{|l|c|c|c|c|c|}
\hline Compound & lung & colon & Breast ER+ & prostate & melanoma \\
\hline (27) diosmin & $>200$ & $>200$ & $>200$ & $>200$ & $>200$ \\
\hline (28) naringin & $>200$ & $>200$ & $>200$ & $>200$ & $>200$ \\
\hline (29) isovitexin & $>200$ & $>200$ & 17 & $>200$ & $>200$ \\
\hline (30) neohesperidin & $>200$ & $>200$ & $>200$ & $>200$ & $>200$ \\
\hline (31) prunin & $>200$ & $>200$ & $>200$ & $>200$ & $>200$ \\
\hline (32) quercetrin & $>200$ & $>200$ & $>200$ & $>200$ & $>200$ \\
\hline (33) isosakuranetin & $>200$ & $>200$ & $>200$ & $>200$ & $>200$ \\
\hline rutinoside & & & & & \\
\hline (34) rutin & $>200$ & $>200$ & $>200$ & $>200$ & $>200$ \\
\hline (35) hesperetin trisaccharide & $>200$ & $>200$ & $>200$ & $>200$ & $>200$ \\
\hline (36) narirutin 4\$-glucoside & $>200$ & $>200$ & $>200$ & $>200$ & $>200$ \\
\hline (37) hesperetin 7-glucoside & 42 & $>200$ & 46 & 86 & 61 \\
\hline (38) hesperidin & 106 & 77 & 51 & 101 & $>200$ \\
\hline (39) neoeriocitrin & 70 & $>200$ & $>200$ & $>200$ & $>200$ \\
\hline (40) rhoifolin & $>200$ & $>200$ & $>200$ & $>200$ & $>200$ \\
\hline (41) neodiosmin & $>200$ & $>200$ & $>200$ & $>200$ & $>200$ \\
\hline (42) margaretin & $>200$ & $>200$ & $>200$ & $>200$ & $>200$ \\
\hline
\end{tabular}

Table 5: Antiproliferative Activities $\left(\mathrm{IC}_{50}\right.$ ) of Flavanone and Flavone Glycosides against Five Human Cancer Cell Lines.

Citrus peel flavonoids against human squamous cell carcinoma

Kandaswami., et al. (1991) reported that in vitro study of citrus flavonoids effects on the growth of a human squamous cell carcinoma cell line (HTB43) demonstrated the health benefit and anticancer effects by examination of four plant flavonoids (taxifolin, nobiletin, quercetin and tangeretin). For 3-7 days, cell cultures were treated with each flavonoid $(2-8 \mu \mathrm{g} / \mathrm{ml})$. 3-(4,5-dimethylthiazol-2-yl)-2,5-diphenyltetrazolium bromide could be utilized for cellular growth to determine the viability of cell by counting cells obtained from a colorimetric assay. The polymethoxyflavonoids quercetin and taxifolin demonstrated no significant prevention at any concentration tested in experiments while analysis showed that other flavonoids such as nobiletin and tangeretin remarkably inhibited cell growth on days 5 and 7 at all concentrations. On day 3 , the cell growth prevention ranged from $70-72 \%$ at $\mu \mathrm{g} / \mathrm{ml}$. while on day 5 , the inhibition observed was $61-88 \%$ at $2-4 \mu \mathrm{g} / \mathrm{ml}$ [19]. 
Flavonoids apigenin and quercetin

Caltagirone., et al. (2000) reported that numerous biological roles have been played by flavonoids containing chemoprevention and tumor growth reduction. In Vivo study was conducted in order to investigate and examine the effects of numerous polyphenols through comparison between their classes, on the metastatic potential and growth of B16-B16 melanoma cells. Quercetin, apigenin, (-) epigallocathechin-3-gallate (EGCG), resveratrol, and the anti-estrogen tamoxifen was compared to administrate intraperitoneal by the introduction of B16-B16 cells into syngeneic rats to inhibit the tumor growth without toxicity in a dose-dependent manner. The result reveals the descending order of potency was EGCG $>$ apigenin $=$ quercetin $=$ tamoxifen $>$ resveratrol $>$ control . Moreover, a significantly potential anti-toxic dose of cisplatin was demonstrated to study inhibitory effect by polyphenols. The introduction of quercetin, apigenin, and tamoxifen (but not EGCG or resveratrol) potentiated resulted in the reduction of a number of B16-BL6 colonies in the lungs as well as decrease the invasion of B16-BL6 cells in vitro, dose-dependent delay of lung colonization. Quercetin and apigenin showed more affectivity as compared to tamoxifen. Furthermore, quercetin and apigenin decrease melanoma growth and invasive and metastatic potential and act as vital tool in the treatment of metastatic melanoma [20].

Flavonoid morin

Tanaka., et al. (1999) reported that flavonoid morin was dietary fed in male F344 rats to treat azoxymethane (AOM)-initiated colorectal carcinogenesis to determine the modifying effect during the initiation and post-initiation stages. Colorectal neoplasms were developed in a group of 55 animals by the injections of 15 $\mathrm{mg} / \mathrm{kg}$ body wt AOM for 3 weeks. The dietary exposure of 500 p.p.m morin for 5 weeks during the initiation feeding and for 28 weeks during the post initiation feeding was carried. The result showed a less reduction of 43 and 29\% in adenocarcinoma in the large intestine of rats fed with AOM combined with diet as compared to the rats provided with AOM alone (75\%). Flavonoid morin caused reduction in the polyamine levels in colorectal mucosa and blood, prohibit the proliferative cell nuclear antigen-positive index in aberrant crypt foci, increased the enzymes glutathione $\mathrm{S}$ transferase and quinone reductase activities in large bowel [21].

\section{Future scope}

This review summarizes the results of currently available data regarding in vivo and in vitro analysis of anti-cancer, anti-inflam- matory and anti-proliferative activities of Citrus peel have multiple active molecular components including flavonoids, Provide opportunities for human clinical trials to deal with preventive and therapeutic effects. Formulation of numerous new products could be proved as effective and beneficial to deal with the fatal disease like cancer.

\section{Bibliography}

1. Cancer C. Taxol - Chemotherapy Drugs - Chemocare (2019).

2. Suzawa M., et al. "In vivo anti-carcinogenic property of a formulated citrus peel extract". Functional Foods in Health and Disease 4.3 (2014): 120-129.

3. Sally Robertson, B. 2019. What are Flavonoids.

4. Ke Z., et al. " $<\mathrm{i}>$ Citrus $</ \mathrm{i}>$ Flavonoids and Human Cancers". Journal of Food and Nutrition Research 3.5 (2015): 341-351.

5. Murakami A., et al. "Inhibitory effect of citrus nobiletin on phorbol ester-induced skin inflammation, oxidative stress, and tumor promotion in mice". Cancer research 60.18 (2000): 5059-5066.

6. Yoshimizu N., et al. "Anti-tumour effects of nobiletin, a citrus flavonoid, on gastric cancer include: antiproliferative effects, induction of apoptosis and cell cycle deregulation". Alimentary pharmacology and therapeutics 20 (2004): 95-101.

7. Kohno H., et al. "Dietary administration of citrus nobiletin inhibits azoxymethane-induced colonic aberrant crypt foci in rats". Life sciences 69 (2001): 901-913.

8. Lin N., et al. "Novel anti-inflammatory actions of nobiletin, a citrus polymethoxy flavonoid, on human synovial fibroblasts and mouse macrophages". Biochemical pharmacology 65.13 (2003): 2065-2071.

9. Tanaka T., et al. "Chemoprevention of azoxymethane-induced rat colon carcinogenesis by the naturally occurring flavonoids, diosmin and hesperid". Carcinogenesis 18.5 (1997): 957-965.

10. Hirano T., et al. "Citrus flavone tangeretin inhibits leukaemic HL-60 cell growth partially through induction of apoptosis with less cytotoxicity on normal lymphocytes". British journal of cancer 72.6 (1995): 1380

11. Pan MHJ., et al. "Tangeretin induces cell-cycle G1 arrest through inhibiting cyclin-dependent kinases 2 and 4 activities as well as elevating Cdk inhibitors p21 and p27 in human colorectal carcinoma cells". Carcinogenesis 23.10 (2002): 1677-1684. 
12. Pan MH., et al. "Inhibition of citrus flavonoids on 12-0-tetradecanoylphorbol 13-acetate-induced skin inflammation and tumorigenesis in mice". Food Science and Human Wellness 1 (2012): 65-73.

13. Suzawa M., et al. "In vivo anti-carcinogenic property of a formulated citrus peel extract". Functional Foods in Health and Disease 4 (2014): 120-129.

14. Lai CS., et al. "Potent anti-cancer effects of citrus peel flavonoids in human prostate xenograft tumors". Food and function 4 (2013): 944-949.

15. Gosslau A., et al. "Anti-Inflammatory Effects of Characterized Orange Peel Extracts Enriched With Bioactive Polymethoxyflavones". Food Science and Human Wellness 3 (2013): 26-35.

16. Lai CS., et al. "Chemoprevention of Colonic Tumorigenesis By Dietary Hydroxylated Polymethoxyflavones In Azoxymethane-Treated Mice". Molecular nutrition and food research 55 (2011): 278-290.

17. Hayashi AA., et al. "Effects of daily oral administration of quercetin chalcone and modified citrus pectin on implanted colon-25 tumor growth in Balb-c mice". Alternative Medicine Review 5 (2000): 546-552.

18. Manthey JA and Guthrie N. "Antiproliferative activities of citrus flavonoids against six human cancer cell lines". Journal of Agricultural and Food Chemistry 50 (2002): 5837-5843.

19. Kandaswami C., et al. "Antiproliferative effects of citrus flavonoids on a human squamous cell carcinoma in vitro". Cancer letters 56 (1991): 147-152.

20. Caltagirone S., et al. "Flavonoids apigenin and quercetin inhibit melanoma growth and metastatic potential". International Journal of Cancer 87 (2000): 595-600.

21. Tanaka T., et al. "Modifying Effects of a Flavonoid Morin On Azoxymethane-Induced Large Bowel Tumorigenesis In Rats". Carcinogenesis 20 (1999): 1477-1484.

Volume 3 Issue 8 August 2019

(C) All rights are reserved by Shahid Mahmood., et al. 